\title{
Measuring dystrophin-faster is not necessarily better
}

\author{
Virginia Arechavala-Gomeza, Lucy Feng, Jennifer E. Morgan and Francesco Muntoni
}

An article in the March issue (A novel imaging method to quantify low levels of dystrophin in Duchenne muscular dystrophy. Nat. Rev. Neurol. 8, 120; 2012) ${ }^{1}$ highlighted findings on a new method for rapid dystrophin quantification in Duchenne muscular dystrophy (DMD). ${ }^{2}$ Although of interest, we believe caution is required in the interpretation of dystrophin measurements obtained using this new technique.

DMD is caused by a deficit of dystrophin protein at the sarcolemma of muscle fibres. ${ }^{3}$ Quantification of dystrophin on muscle biopsies is the main diagnostic test for DMD when genetic testing is unavailable. Restoration of dystrophin expression is the aim of several therapeutic approaches such as redirection of splicing with antisense oligonucleotides, ${ }^{4,5}$ gene therapy, ${ }^{6}$ stem cell therapy, ${ }^{7}$ and nonsense mutation readthrough. ${ }^{8,9}$ Precise quantification of dystrophin protein both before and after treatment is crucial to evaluate the biochemical success of therapeutic interventions for DMD.

Until recently, counting dystrophinpositive fibres or western blotting were the only quantitative methods available, but researchers preparing for the first trials developed a method to sensitively quantify dystrophin and other associated proteins in the muscle fibre sarcolemma using only two muscle sections per antibody. ${ }^{10}$ The method uses intensity measurements from fluorescently labelled dystrophin antibodies with spectrin labelling as a normalizing factor. The technique greatly advanced dystrophin quantification owing to its sensitivity, requirement for very little sample, capacity to confirm the localization of the protein at the sarcolemma, and accessibility to most pathology laboratories. Despite being labour-intensive, this method has been used in the analysis of several clinical trials,,${ }^{4,5}$ and in human ${ }^{11-13}$ and mouse ${ }^{13,14}$ studies.

Aided by a new spectrin antibody that enabled immunostaining for dystrophin and spectrin on the same section, researchers have been able to automate this technique, ${ }^{2}$
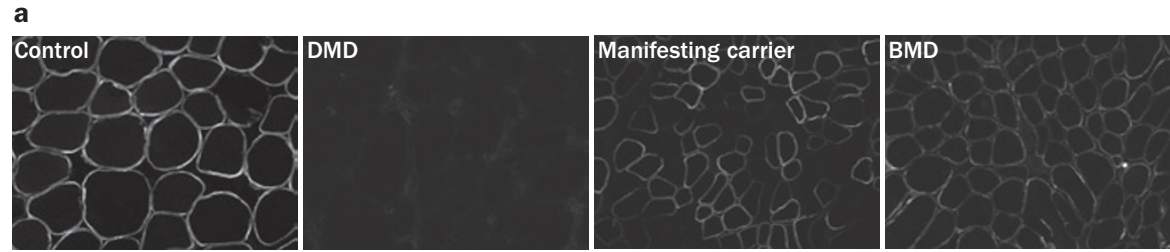

b
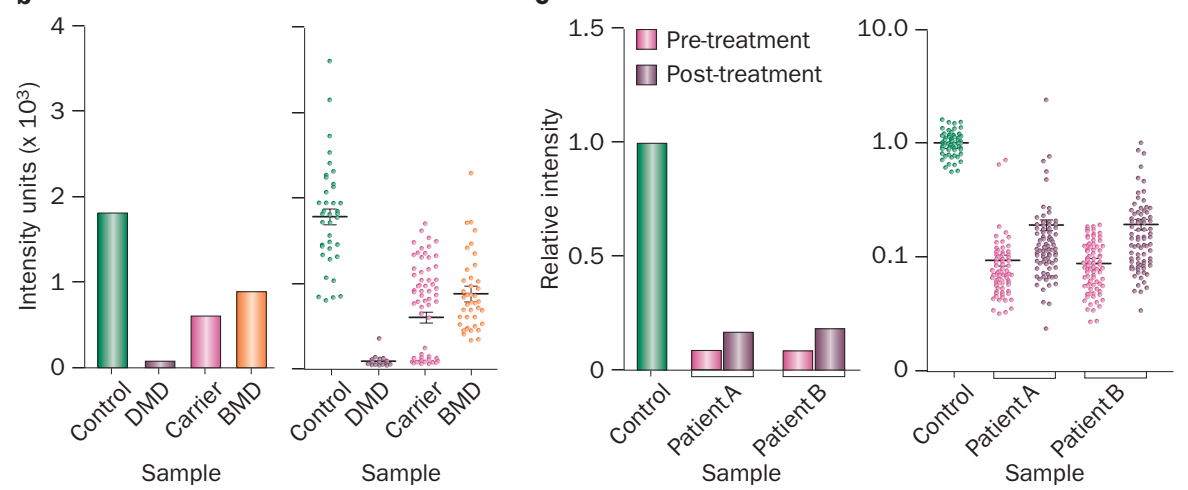

Figure 1 | Comparison of methods for dystrophin quantification. a | Transverse cryosections of quadriceps muscle biopsies, immunostained with a dystrophin antibody. b | Intensity profiles of images in panel a, captured as an average measurement ${ }^{2}$ (left) or with multiple measurements ${ }^{10}$ (right). c | Two samples from a recent systemic clinical ${ }^{4}$ trial analysed using average measurement (left) and multiple measurement (right). Abbreviations: BMD, Becker muscular dystrophy; DMD; Duchenne muscular dystrophy.

which should accelerate the analysis of muscle biopsies in ongoing clinical trials. Despite the unequivocal advance that this method entails, one should note the potential drawbacks. One important aspect is that the original method involves collection of up to 40 data points per section, each corresponding to a muscle fibre, whereas the recent modification records average dystrophin intensity for the whole image. In a manifesting carrier, the clear segregation of positive and negative dystrophin measurements that is evident using the original method ${ }^{10}$ is lost with the new 'averaging' method (Figure 1a,b). ${ }^{2}$ Similarly, two patients from a recent clinical trial have almost identical levels of dystrophin when assessed using the new method, whereas the original method shows that the average of one sample is skewed owing to a few high measurements (Figure 1c).
Some patients have a few intensely dystrophin-positive fibres, whereas others have more dimly staining dystrophinpositive fibres, ${ }^{4}$ which accounts for the variability in dystrophin expression in these trials. To guarantee optimal evaluation of the response to treatment, it is vital that the maximum amount of information is collected from the small samples available. As the two image-capture methods do not differ, ${ }^{2,10}$ only a slight modification of the method to include several average measurements per image would suffice.

The Dubowitz Neuromuscular Centre, University College London Institute of Child Health, 30 Guilford Street, London WC1N 1EH, UK (V. Arechavala-Gomeza, L. Feng, J. E. Morgan, F. Muntoni).

Correspondence to: V. Arechavala-Gomeza v.arechavala@ucl.ac.uk

doi:10.1038/nrneurol.2012.15-c1 
Competing interests

The authors declare no competing interests.

1. Malpass, K. A novel imaging method to quantify low levels of dystrophin in Duchenne muscular dystrophy. Nat. Rev. Neurol. 8, 120 (2012).

2. Taylor, L. E., Kaminoh, Y. J., Rodesch, C. K. \& Flanigan, K. M. Quantification of dystrophin immunofluorescence in dystrophinopathy muscle specimens. Neuropathol. Appl. Neurobiol. http://dx.doi.org/10.1111/ j.1365-2990.2012.01250.x.

3. Hoffman, E. P., Brown, R. H. Jr \& Kunkel, L. M. Dystrophin: the protein product of the Duchenne muscular dystrophy locus. Cell 51, 919-928 (1987).

4. Cirak, S. et al. Exon skipping and dystrophin restoration in patients with Duchenne muscular dystrophy after systemic phosphorodiamidate morpholino oligomer treatment: an open-label, phase 2, dose-escalation study. Lancet $\mathbf{3 7 8}$, 595-605 (2011).

5. Kinali, M. et al. Local restoration of dystrophin expression with the morpholino oligomer AVI-4658 in Duchenne muscular dystrophy: a single-blind, placebo-controlled, doseescalation, proof-of-concept study. Lancet Neurol. 8, 918-928 (2009).

6. Mendell, J. R. et al. Dystrophin immunity in Duchenne's muscular dystrophy. N. Engl. J. Med. 363, 1429-1437 (2010).

7. Patel, K. \& Morgan, J. $185^{\text {th }}$ ENMC International Workshop: stem/precursor cells as a therapeutic strategy for muscular dystrophies 3-5 June 2011, Naarden, The Netherlands. Neuromuscul. Disord. 22, 447-452 (2012).

8. Hirawat, S. et al. Safety, tolerability, and pharmacokinetics of PTC124, a nonaminoglycoside nonsense mutation suppressor, following single- and multiple-dose administration to healthy male and female adult volunteers. J. Clin. Pharmacol. 47, 430-444 (2007).

9. Malik, V. et al. Gentamicin-induced readthrough of stop codons in Duchenne muscular dystrophy. Ann. Neurol. 67, 771-780 (2010).

10. Arechavala-Gomeza, V. et al. Immunohistological intensity measurements as a tool to assess sarcolemma-associated protein expression. Neuropathol. Appl. Neurobiol. 36, 265-274 (2010).

11. Arechavala-Gomeza, V. et al. Revertant fibres and dystrophin traces in Duchenne muscular dystrophy: implication for clinical trials. Neuromuscul. Disord. 20, 295-301 (2010).

12. Cirak, S. et al. Restoration of the dystrophinassociated glycoprotein complex after exon skipping therapy in Duchenne muscular dystrophy. Mol. Ther. 20, 462-467 (2011).

13. Anthony, K. et al. Dystrophin quantification and clinical correlations in Becker muscular dystrophy: implications for clinical trials. Brain 134, 3547-3459 (2011).

14. Malerba, A. et al. Chronic systemic therapy with low-dose morpholino oligomers ameliorates the pathology and normalizes locomotor behavior in mdx mice. Mol. Ther. 19, 345-354 (2011).

15. Malerba, A., Boldrin, L. \& Dickson, G. Long-term systemic administration of unconjugated morpholino oligomers for therapeutic expression of dystrophin by exon skipping in skeletal muscle: implications for cardiac muscle integrity. Nucleic Acid Ther. 21 293-298 (2011). 